\title{
Shot noise in out-of equilibrium resonant tunneling through an Anderson impurity
}

\author{
Guo-Hui Ding and Tai-Kai Ng \\ Department of Physics, The Hong Kong University of Science and Technology, Clear Water Bay, Kowloon, Hong Kong, China
}

(Received 28 August 1997)

\begin{abstract}
The noise characteristics of a single Anderson impurity coupled to two leads at different chemical potential is studied using the equation of motion method. We find that the current noise spectral density has an upturn as the frequency matches the chemical potential difference at low temperature. The low-frequency noise shows resonant behavior at equilibrium at low temperature because of the Kondo effect, and is suppressed significantly due to the Coulomb blockade effect on the impurity site when $\Delta \mu \gg T \sim T_{K}$. [S0163-1829(97)50848-4]
\end{abstract}

At present there is much interest in the study of electronic transport through quantum dots. For small dots with Coulomb repulsive interaction $U$ and energy level spacing $\Delta \epsilon \gg k T$, the system can be described by the single impurity Anderson model. At equilibrium, due to the on-site Coulomb repulsion, the Kondo effect emerges at low temperature. ${ }^{1}$ The problem of the nonequilibrium Kondo effect has only been investigated carefully in recent years. ${ }^{2-7}$ Theoretical calculations show that for a dot coupled to two leads, a resonant peak of differential conductance emerges as a result of the Kondo effect, and the density of states on the dot site has a peak pinned at the chemical potential between the leads. For a finite chemical potential difference of the two leads, the peak of the density of states splits into two peaks pinned at the chemical potential of each lead. The two-peak structure in the electron spectral function is difficult to probe by dc $I-V$ measurements, but can be probed by imposing an ac bias voltage on the dc bias of the leads. ${ }^{8}$

The electrical current through the quantum dot is fluctuating in time even under a dc bias. Its fluctuation is characterized by the current noise spectral density. The noise characteristic of this quantum device is important and interesting from both technical and theoretical viewpoints, and it has also been investigated extensively both theoretically and experimentally. ${ }^{9-15}$ Theoretically, these investigations of noise spectra have been restricted to a noninteracting system or by Hartree approximation to include the on-site Coulomb repulsion, and cannot probe the low-temperature properties of the system. In the linear response region, the noise spectral density is related to the ac conductance by the Einstein relation $S\left(\omega_{0}\right)=4 k T \sigma\left(\omega_{0}\right)$, and contains the same information as the ac conductance. Away from equilibrium, the fluctuation dissipation theorem is not satisfied, and the noise spectrum is not directly related to the linear response conductance and has to be computed separately. In this paper we shall study the current noise of this system based on the infinite- $U$ Anderson model. We shall only consider the fluctuation of the average current $I(t)=\left[I_{L}(t)-I_{R}(t)\right] / 2$ for simplicity. Since the noise spectral density $S\left(\omega_{0}\right)$ is given by the Fourier transform of the correlation function $S\left(t-t^{\prime}\right)=\left\langle\delta \hat{I}(t) \delta \hat{I}\left(t^{\prime}\right)+\delta \hat{I}\left(t^{\prime}\right) \delta \hat{I}(t)\right\rangle$, we must calculate a nonequilibrium current-current correlation function. To do that we first study the response of the current $I_{\eta}(t)$ to an applied external potential $V_{\eta^{\prime}}\left(t^{\prime}\right)$ on the lead, which gives the retarded Green function of the current operator and the occupation number operator of the lead,

$$
\widetilde{S}\left(t-t^{\prime}\right)=\frac{\delta I_{\eta}(t)}{\delta V_{\eta^{\prime}}\left(t^{\prime}\right)}=-i \theta\left(t-t^{\prime}\right)<\left[\hat{I}_{\eta}(t), \hat{N}_{\eta^{\prime}}\left(t^{\prime}\right)\right] .
$$

By the time-loop method of perturbation theory, the response function could be separated into two parts with the time variable $t^{\prime}$ on either the positive or the negative branch of the time loop, and the part with the time variable $t^{\prime}$ on the negative branch will give the correlation function $\left\langle\hat{N}_{\eta^{\prime}}\left(t^{\prime}\right) \hat{I}_{\eta}(t)\right\rangle$. Due to the simple form of the function $\widetilde{S}$ in our theory, we can separate explicitly this part that contributes to the correlation function $\left\langle\hat{N}_{\eta^{\prime}}\left(t^{\prime}\right) \hat{I}_{\eta}(t)\right\rangle$ in the response function. The current-current correlation function $\left\langle\hat{I}_{\eta^{\prime}}\left(t^{\prime}\right) \hat{I}_{\eta}(t)\right\rangle$ is obtained by differentiating the number occupation operator $\hat{N}\left(t^{\prime}\right)$ with respect to $t^{\prime}$.

We consider the system described by the following timedependent Hamiltonian,

$$
\begin{aligned}
H= & \sum_{k, \eta, \sigma} \epsilon_{k \eta}(t) c_{k \eta \sigma}^{\dagger} c_{k \eta \sigma}+\sum_{k, \eta, \sigma}\left[t_{\eta} c_{k \eta \sigma}^{\dagger} d_{\sigma}+\text { H.c. }\right] \\
& +\sum_{\sigma} \epsilon_{0} d_{\sigma}^{\dagger} d_{\sigma}+U n_{d \uparrow} n_{d \downarrow},
\end{aligned}
$$

where $\eta=L, R$ are the lead indices, $c_{k \eta \sigma}^{\dagger}\left(c_{k \eta \sigma}\right)$ creates (destroys) an electron in lead $\eta$ with spin $\sigma$, and $d_{\sigma}^{\dagger}\left(d_{\sigma}\right)$ are creation and annihilation operators on the quantum dot. The second term in the Hamiltonian describes the hopping between the leads and the dot, while the last term represents the Coulomb interaction between the electrons on the dot site for which we shall finally take the limit $U \rightarrow \infty$. The timedependent single-particle energy $\epsilon_{k \eta}(t)$ is given by $\epsilon_{k \eta}(t)=\epsilon_{k}+V_{\eta} \cos \left(\omega_{0} t\right)$, with the $V_{\eta}$ term reflects the ac bias, and $\epsilon_{0}$ is the energy level on the quantum dot. The electrons in the leads are assumed to be in their own equilibrium states separately with chemical potentials $\mu_{L}$ and $\mu_{R}$.

The current flows from the lead $\eta$ to the central dot can be calculated from the time evolution of the occupation number operator of the lead, 


$$
\begin{aligned}
I_{\eta}(t) & =-e\left\langle\frac{d \hat{N}_{\eta}}{d t}\right\rangle=i e \sum_{k, \sigma}\left[t_{\eta}\left\langle c_{k \eta \sigma}^{\dagger} d_{\sigma}\right\rangle-t_{\eta}^{*}\left\langle d_{\sigma}^{\dagger \dagger} c_{k \eta \sigma}\right\rangle\right] \\
& =e \sum_{k, \sigma}\left[t_{\eta} G_{d, k \eta \sigma}^{<}(t, t)-t_{\eta}^{*} G_{k \eta \sigma, d}^{<}(t, t)\right],
\end{aligned}
$$

where we have used the definition of the Keldysh Green function $\left.G_{d, k \eta \sigma}^{<}\left(t, t^{\prime}\right) \equiv i\left\langle c_{k \eta \sigma}^{\dagger} t^{\prime}\right) d_{\sigma}(t)\right\rangle$ and $G_{k \eta \sigma, d}^{<}\left(t, t^{\prime}\right)$ $\left.\equiv i\left\langle d_{\sigma}^{\dagger} t^{\prime}\right) c_{k \eta \sigma}(t)\right\rangle$. The average current from the left lead to right lead is given by $I(t)=\left[I_{L}(t)-I_{R}(t)\right] / 2$. Since the electrons in the leads are noninteracting, it is easy to obtain the Dyson equations, ${ }^{16}$

$$
\begin{aligned}
G_{d, k \eta \sigma}^{<}\left(t, t^{\prime}\right)= & \int d t_{1} t_{\eta}^{*}\left[G_{d \sigma}^{r}\left(t, t_{1}\right) g_{k \eta}^{<}\left(t_{1}, t^{\prime}\right)\right. \\
& \left.+G_{d \sigma}^{<}\left(t, t_{1}\right) g_{k \eta}^{a}\left(t_{1}, t^{\prime}\right)\right] \\
G_{k \eta \sigma, d}^{<}\left(t, t^{\prime}\right)= & \int d t_{1} t_{\eta}\left[g_{k \eta}^{r}\left(t, t_{1}\right) G_{d \sigma}^{<}\left(t_{1}, t^{\prime}\right)\right. \\
& \left.+g_{k \eta}^{<}\left(t, t_{1}\right) G_{d \sigma}^{a}\left(t_{1}, t^{\prime}\right)\right],
\end{aligned}
$$

where $G_{d \sigma}^{r}\left(t, t^{\prime}\right)$ and $G_{d \sigma}^{<}\left(t, t^{\prime}\right)$ are the retarded Green function and the Keldysh Green function of the impurity site electron, respectively. In order to obtain the time-dependent behaviors of the current we need to study $G_{d \sigma}^{r}\left(t, t^{\prime}\right)$ and $G_{d \sigma}^{<}\left(t, t^{\prime}\right)$ in the presence of external ac field and on-site Coulomb interaction. This problem has been carried out in Ref. 8 by the equation of motion approach. We first consider the retarded Green function $G_{d \sigma}^{r}\left(t, t^{\prime}\right)$. The equation of motion method consists of differentiating the Green function $G_{d \sigma}^{r}$ with respect to time, thereby generating high-order Green functions that have to be truncated to close the equation of $G_{d \sigma}^{r}\left(t, t^{\prime}\right)$. For the Anderson impurity model with ac field, we introduce the Fourier transform $G\left(\omega, \omega^{\prime}\right)=\int d t \int d t^{\prime} e^{i \omega t} e^{-i \omega^{\prime} t^{\prime}} G\left(t, t^{\prime}\right)$; in the limit $U \rightarrow \infty$ we obtain

$$
\begin{aligned}
\left(\omega-\epsilon_{0}\right) G_{d \sigma}^{r}\left(\omega, \omega^{\prime}\right)= & 2 \pi \delta\left(\omega-\omega^{\prime}\right)\left(1-\left\langle n_{d \bar{\sigma}}\right\rangle\right) \\
& +\int \frac{d \omega^{\prime \prime}}{2 \pi} \Sigma^{r}\left(\omega, \omega^{\prime \prime}\right) G_{d \sigma}^{r}\left(\omega^{\prime \prime}, \omega^{\prime}\right),
\end{aligned}
$$

where the factor $1-\left\langle n_{d \bar{\sigma}}\right\rangle$ goes to $1 / 2$ in the Kondo limit $\epsilon_{0} \ll \mu_{L}, \mu_{R} . \Sigma^{r}\left(\omega, \omega^{\prime}\right)$ is the Fourier transform of the selfenergy $\Sigma^{r}\left(t, t^{\prime}\right)$ that comes from the tunneling of electrons from the impurity site to the leads and the on-site interaction;

$$
\Sigma^{r}\left(t, t^{\prime}\right)=\sum_{\eta} \Gamma_{\eta} \int_{-D}^{D} \frac{d \epsilon_{k}}{2 \pi}\left[1+n_{\eta}\left(\epsilon_{k}\right)\right] g_{k \eta}^{r}\left(t, t^{\prime}\right)
$$

where $\Gamma_{\eta}=2 \pi \rho(0)\left|t_{\eta}\right|^{2}$, with $\rho(0)$ the density of states in the two leads. $D$ is the bandwidth, and $g_{k \eta}^{r}\left(t, t^{\prime}\right)$ is the timedependent retarded Green function in the leads $\eta$ for free electrons,

$$
\begin{aligned}
g_{k \eta}^{r}\left(t, t^{\prime}\right)= & -i \theta\left(t-t^{\prime}\right) \\
& \times \exp \left(-i \epsilon_{k}\left(t-t^{\prime}\right)-i \int_{t^{\prime}}^{t} d t_{1} V_{\eta} \cos \left(\omega_{0} t_{1}\right)\right) .
\end{aligned}
$$

By the formal Keldysh Green-function technique it is easy to prove that $G_{d \sigma}^{<}\left(t, t^{\prime}\right)=\int d t_{1} \int d t_{2} G_{d \sigma}^{r}\left(t, t_{1}\right) \Sigma^{<}\left(t_{1}, t_{2}\right)$ $G_{d \sigma}^{a}\left(t_{2}, t^{\prime}\right)$, where $\Sigma^{<}$is the Keldysh self-energy. Assuming that the operational rules given by Langreth ${ }^{17}$ for contour integration is satisfied, and making a similar approximation as in the calculation of the retarded Green function $G^{r}$, we obtain also

$$
\begin{aligned}
\Sigma^{<}\left(t, t^{\prime}\right)= & 2 i \sum_{\eta} \int \frac{d \epsilon_{k}}{2 \pi} \Gamma_{\eta}\left(1+n_{\eta}\left(\epsilon_{k}\right)\right) n_{\eta}\left(\epsilon_{k}\right) \\
& \times \exp \left(-i \epsilon_{k}\left(t-t^{\prime}\right)-i \int_{t^{\prime}}^{t} d t_{1} V_{\eta} \cos \left(\omega_{0} t_{1}\right)\right) .
\end{aligned}
$$

To calculate the response function $\widetilde{S}$, we expand the Green functions in the formula of current to first-order term of the external ac field. Using the identities $G_{d, k \eta \sigma}^{<}=G_{d, k \eta \sigma}^{t}-G_{d, k \eta \sigma}^{r}$ and $G_{k \eta \sigma, d}^{<}=G_{k \eta \sigma, d}^{t}-G_{k \eta \sigma, d}^{r}$, we can show that $\widetilde{S}$ is given by

$$
\begin{aligned}
\widetilde{S}\left(t-t^{\prime}\right)= & e \sum_{k \sigma}\left\{\delta _ { \eta , \eta ^ { \prime } } \left[t_{\eta} G_{d, k \eta \sigma}^{t}\left(t-t^{\prime}\right) g_{k \eta^{\prime}}^{t}\left(t^{\prime}-t\right)\right.\right. \\
& \left.-t_{\eta}^{*} g_{k \eta^{\prime}}^{t}\left(t-t^{\prime}\right) G_{k \eta \sigma, d}^{t}\left(t^{\prime}-t\right)\right]+\sum_{k^{\prime}} 2[1 \\
& \left.+n_{\eta^{\prime}}\left(\epsilon_{k^{\prime}}\right)\right]\left[t_{\eta} G_{d, k^{\prime} \eta^{\prime} \sigma}^{t}\left(t-t^{\prime}\right) \Pi_{k^{\prime} \eta^{\prime}, k \eta}^{t}\left(t^{\prime}-t\right)\right. \\
& \left.\left.-t_{\eta}^{*} \Pi_{k \eta, k^{\prime} \eta^{\prime}}^{t}\left(t-t^{\prime}\right) G_{k^{\prime} \eta^{\prime} \sigma, d}^{t}\left(t^{\prime}-t\right)\right]\right\} \\
& -e \sum_{k \sigma}\left\{\delta _ { \eta , \eta ^ { \prime } } \left[t_{\eta} G_{d, k \eta \sigma^{\prime}}^{<}\left(t-t^{\prime}\right) g_{k \eta^{\prime}}^{>}\left(t^{\prime}-t\right)\right.\right. \\
& \left.-t_{\eta}^{*} g_{k \eta^{\prime}}^{<}\left(t-t^{\prime}\right) G_{k \eta \sigma, d}^{>}\left(t^{\prime}-t\right)\right]+\sum_{k^{\prime}} 2[1 \\
& \left.+n_{\eta^{\prime}}\left(\epsilon_{k^{\prime}}\right)\right]\left[t_{\eta} G_{d, k^{\prime} \eta^{\prime} \sigma}^{<}\left(t-t^{\prime}\right) \Pi_{k^{\prime} \eta^{\prime}, k \eta^{\prime}}^{>}\left(t^{\prime}-t\right)\right. \\
& \left.\left.-t_{\eta}^{*} \Pi_{k \eta, k^{\prime} \eta^{\prime}}^{<}\left(t-t^{\prime}\right) G_{k^{\prime} \eta^{\prime} \sigma, d}^{>}\left(t^{\prime}-t\right)\right]\right\}
\end{aligned}
$$

where $\Pi^{<}, \Pi^{>}$and $\Pi^{t}$ are elements of the $2 \times 2$ matrix Green function $\Pi$, which is defined as a contour integration, ${ }^{16}$

$$
\begin{aligned}
\Pi_{k \eta, k^{\prime} \eta^{\prime}}\left(t-t^{\prime}\right)= & t t_{\eta} t^{*} \int_{C} \int_{C} d t_{1} d t_{2} g_{k \eta}\left(t-t_{1}\right) G_{d \sigma}\left(t_{1}-t_{2}\right) \\
& \times g_{k^{\prime} \eta^{\prime}}\left(t_{2}-t^{\prime}\right)
\end{aligned}
$$

By making a comparison with the noninteracting system, we can see that the terms in the second pair of curly brackets in 
Eq. (8) come from the integration of time variable $t^{\prime}$ on the negative branch; hence they will give the correlation function $\left\langle\hat{N}_{\eta^{\prime}}\left(t^{\prime}\right) \hat{I}_{\eta}(t)\right\rangle$. We can differentiate the number operator $\hat{N}_{\eta^{\prime}}\left(t^{\prime}\right)$ to obtain the correlation function of the current
$\left\langle\hat{I}_{\eta^{\prime}}\left(t^{\prime}\right) \hat{I}_{\eta}(t)\right\rangle$, and finally calculate the noise spectral density. For simplicity we consider the system with symmetric structure $\Gamma_{L}=\Gamma_{R}=\Gamma$. After the Fourier transformation the current noise spectral density is given by

$$
\begin{aligned}
S\left(\omega_{0}\right)= & \frac{1}{2} e^{2} \int \frac{d \omega}{2 \pi}\left\{i \Gamma\left[n_{L}(\omega)+n_{R}(\omega)\right]\left[G^{r}\left(\omega+\omega_{0}\right)-G^{a}\left(\omega+\omega_{0}\right)+G^{<}\left(\omega+\omega_{0}\right)\right]+i \Gamma\left[n_{L}\left(\omega+\omega_{0}\right)+n_{R}\left(\omega+\omega_{0}\right)\right] G^{<}(\omega)\right. \\
& -2 G^{<}(\omega)+\Gamma^{2}\left[n_{L}(\omega)-n_{R}(\omega)\right]\left[n_{L}\left(\omega+\omega_{0}\right)-n_{R}\left(\omega+\omega_{0}\right)\right]\left[2+n_{L}(\omega)+n_{R}(\omega)+n_{L}\left(\omega+\omega_{0}\right)+n_{R}\left(\omega+\omega_{0}\right)\right] \\
& \times\left[G^{r}(\omega) G^{r}\left(\omega+\omega_{0}\right)+G^{a}(\omega) G^{a}\left(\omega+\omega_{0}\right)\right]+\left(i \Gamma [ n _ { L } ( \omega ) - n _ { R } ( \omega ) ] \left\{\left[\Sigma_{L}\left(\omega+\omega_{0}\right)-\Sigma_{L}(\omega)-\Sigma_{R}\left(\omega+\omega_{0}\right)\right.\right.\right. \\
& \left.+\Sigma_{R}(\omega)\right] G^{r}(\omega) G^{r}\left(\omega+\omega_{0}\right)+\left[\Sigma_{L}^{*}\left(\omega+\omega_{0}\right)-\Sigma_{L}(\omega)-\Sigma_{R}^{*}\left(\omega+\omega_{0}\right)+\Sigma_{R}(\omega)\right]\left[G^{r}(\omega) G^{<}\left(\omega+\omega_{0}\right)-G^{r}(\omega) G^{a}(\omega\right. \\
& \left.\left.\left.\left.+\omega_{0}\right)\right]\right\}+ \text { H.c. }\right)+\left\{-i \Gamma\left[n_{L}\left(\omega+\omega_{0}\right)-n_{R}\left(\omega+\omega_{0}\right)\right]\left[\Sigma_{L}^{*}\left(\omega+\omega_{0}\right)-\Sigma_{L}(\omega)-\Sigma_{R}^{*}\left(\omega+\omega_{0}\right)+\Sigma_{R}(\omega)\right] G^{<}(\omega) G^{a}(\omega\right. \\
& \left.\left.\left.+\omega_{0}\right)+ \text { H.c. }\right\}\right\}+\left\{\omega_{0} \rightarrow-\omega_{0}\right\},
\end{aligned}
$$

where $G^{r}(\omega)$ is the retarded Green function on the central site without small ac bias field. The spin indices have been omitted because the results are independent of whether the spin is up or down. We obtain

$$
G^{r}(\omega)=\frac{1 / 2}{\omega-\epsilon_{0}+i \Gamma-\Sigma(\omega)},
$$

where $\Sigma(\omega)=\Sigma_{L}(\omega)+\Sigma_{R}(\omega)$, with

$$
\Sigma_{\eta}(\omega)=\Gamma_{\eta} \int_{-D}^{D} \frac{d \epsilon}{2 \pi} \frac{n_{\eta}(\epsilon)}{\omega-\epsilon+i \delta} .
$$

The Keldysh Green function $G^{<}(\omega)$ is given by

$$
G^{<}(\omega)=\sum_{\eta} 2 i n_{\eta}(\omega)\left[1+n_{\eta}(\omega)\right] G^{r}(\omega) G^{a}(\omega)
$$

First we consider the system in equilibrium $\mu_{L}=\mu_{R}$, where the Fermi distribution functions in the leads $n_{L}(\omega)=n_{R}(\omega)=n(\omega)$. The formula for the noise spectral density can be greatly simplified, where

$$
\begin{aligned}
S\left(\omega_{0}\right)= & i e^{2} \Gamma \int \frac{d \omega}{2 \pi}\left(\left\{n ( \omega ) \left[G^{r}\left(\omega+\omega_{0}\right)-G^{a}\left(\omega+\omega_{0}\right)\right.\right.\right. \\
& \left.\left.+G^{<}\left(\omega+\omega_{0}\right)\right]+n\left(\omega+\omega_{0}\right) G^{<}(\omega)-2 G^{<}(\omega)\right\} \\
& \left.+\left[\omega_{0} \rightarrow-\omega_{0}\right]\right) .
\end{aligned}
$$

By the fluctuation-dissipation theorem, $S\left(\omega_{0}\right)$ is related to the linear conductance $\sigma\left(\omega_{0}\right)$,

$$
S\left(\omega_{0}\right)=\frac{e^{\beta \omega_{0}}+1}{e^{\beta \omega_{0}-1}} 2 \omega_{0} \sigma\left(\omega_{0}\right) .
$$

For frequency much larger than the temperature $\left(\omega_{0} \gg 1 / \beta\right)$, the noise spectral density is proportional to the frequency $S\left(\omega_{0}\right)=2 \omega_{0} \sigma\left(\omega_{0}\right)$. In the limit $\omega_{0} \ll 1 / \beta$, it gives the wellknown Einstein relation $S\left(\omega_{0}\right)=4 T \sigma\left(\omega_{0}\right)$. For temperature lower than the Kondo temperature, the noise spectral density increases at low frequency due to the Kondo resonance in the conductance. This phenomenon can be shown more clearly as we consider the zero-frequency noise. In Fig. 1 we show the temperature dependence of the zero-frequency noise. We have chosen parameters $D=100, \Gamma_{L}=\Gamma_{R}=1.0$, and $\epsilon_{0}=-4.5$ in our calculation. The Kondo temperature $T_{K}$ is equal to 0.0017 for these parameters. We see that there is a peak in $S\left(\omega_{0}=0\right) / T$ as the temperature decreases for this infinite on-site interacting system. This effect is qualitatively the same for the frequency $\omega_{0} \leqslant T_{K}$. We notice that for the noninteracting system $S\left(\omega_{0}=0\right) / T$ is almost temperature independent.

The noise spectral density away from equilibrium can also be calculated in our approximation, and the results are shown in Figs. 2 and 3. In Fig. 2 we show the noise spectral density $S\left(\omega_{0}\right)$ as a function of the frequency $\omega_{0}$ at fixed temperature $T=T_{K}$ for three different chemical potential differences of the leads, $\Delta \mu=2 T_{K}, 20 T_{K}$, and $50 T_{K}$. For the chemical potential difference $\Delta \mu$ much larger than the Kondo temperature $T_{K}$, there is a turn of the $S\left(\omega_{0}\right)$ at frequency $\omega_{0}=\Delta \mu$. This effect also presents in the noninteracting system and is a result of the finite chemical potential difference between the leads. For $\Delta \mu \approx T$, this structure in the noise

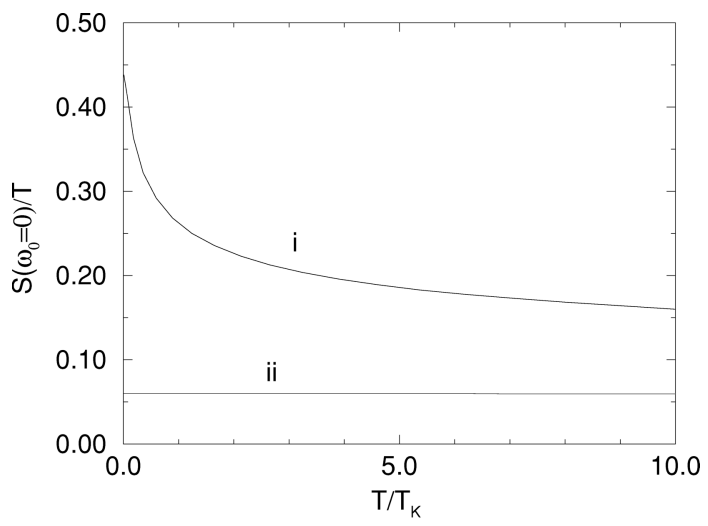

FIG. 1. The zero-frequency noise at equilibrium as a function of temperature for an infinite on-site interaction system (i), and a noninteracting system (ii). 


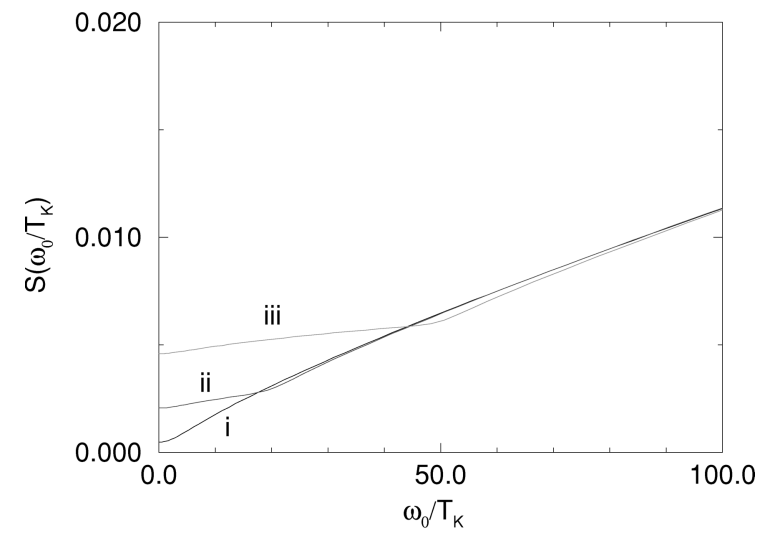

FIG. 2. The noise spectral density at fixed temperature $T=T_{K}$ for three different chemical potential differences $\Delta \mu=2 T_{k}$ (i), $20 T_{k}$ (ii), $50 T_{K}$ (iii).

spectral density disappears, since the noise of thermal excitation becomes more important than the noise caused by the chemical potential difference. In Fig. 3 we show the zerofrequency noise $S(0)$ as a function of the current $I$ at fixed temperature $T=T_{K}$ for the interacting and noninteracting systems. For the system without on-site Coulomb repulsion interaction, the shot noise is almost full for the parameters we use, which agrees with the relation between the zerofrequency noise and the transmission probability deduced in Ref. 13, since in this case the transmission probability is much less than one. For the interacting system, we see that the short noise is significantly suppressed. This indicates the strong correlation of the electrons on the dot site.

In summary, using the equation of motion method we have obtained the noise characteristics of the system with an

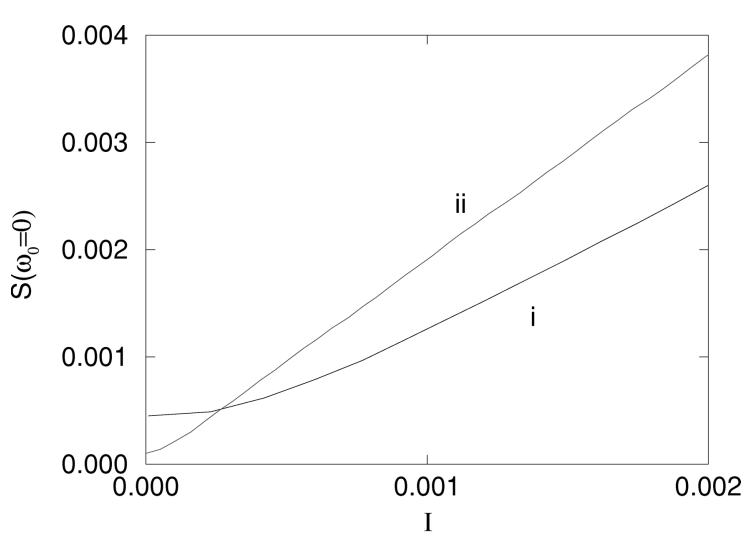

FIG. 3. The zero-frequency noise as a function of the current at temperature $T=T_{K}$ for an interacting (i) and a noninteracting system (ii).

Anderson impurity coupled to leads in both the equilibrium and the nonequilibrium case. We note that the equation of motion approach gives only qualitatively correct behavior, 5,8 and our result can only be trusted qualitatively. The lowfrequency noise shows resonant behavior in the Kondo regime, and the shot noise is suppressed due to the Coulomb blockade effect when $\Delta \mu \gg T$. In this paper we have considered that the energy level $\epsilon_{0}$ of the dot site is far below the Fermi energies in the leads. As the energy level $\epsilon_{0}$ varies, the noise characteristics may change dramatically. The case with $\epsilon_{0}$ located nearby the Fermi surfaces of the leads is also interesting and will be investigated in a later publication, in which the detail results of the various correlation functions $\left\langle I_{\eta}(t) I_{\eta^{\prime}}\left(t^{\prime}\right)\right\rangle$ will also be presented.

This work was supported by HKUGC through RGC Grant No. HKUST 646/94P.
${ }^{1}$ K. G. Wilson, Rev. Mod. Phys. 47, 773 (1975).

${ }^{2}$ D. C. Ralph and R. A. Buhrman, Phys. Rev. Lett. 72, 3401 (1994)

${ }^{3}$ D. Goldhaber-Gordon, H. Shtrikman, D. Mahalu, D. AbuschMagder, U. Meirav, and M. A. Kastner (unpublished).

${ }^{4}$ S. Hershfield, J. H. Davies, and J. W. Wilkins, Phys. Rev. Lett. 67, 3720 (1991).

${ }^{5}$ Y. Meir and N. S. Wingreen, Phys. Rev. Lett. 68, 2512 (1992).

${ }^{6}$ Y. Meir, N. S. Wingreen, and P. A. Lee, Phys. Rev. Lett. 66, 3048 (1991); ibid. 70, 2601 (1993).

${ }^{7}$ T. K. Ng and P. A. Lee, Phys. Rev. Lett. 61, 1768 (1988).

${ }^{8}$ T. K. Ng, Phys. Rev. Lett. 76, 487 (1996).

${ }^{9}$ L. Y. Chen and C. S. Ting, Phys. Rev. B 43, 4534 (1991).

${ }^{10}$ S. Hershfield, Phys. Rev. B 46, 7061 (1992).
${ }^{11}$ B. Yurke and G. P. Kochanski, Phys. Rev. B 41, 8184 (1990).

${ }^{12}$ M. Buttiker, Phys. Rev. Lett. 65, 2901 (1990).

${ }^{13}$ C. W. J. Beenakker and H. van Houten, Phys. Rev. B 43, 12066 (1991).

${ }^{14}$ Th. Martin and R. Landauer, Phys. Rev. B 45, 1742 (1992).

${ }^{15}$ Y. P. Li, A. Zaslavsky, D. C. Tsui, M. Santos, and M. Shayegan, Phys. Rev. B 41, 8388 (1990).

${ }^{16}$ A. P. Jauho, N. S. Wingreen, and Y. Meir, Phys. Rev. B 50, 5528 (1994).

${ }^{17}$ D. C. Langreth, in Linear and Nonlinear Electron Transport in Solids, Vol. 17 of Nato Advanced Study Institute, Series B: Physics, edited by J. T. Devreese and V. E. Van Doren (Plenum, New York, 1976). 